\title{
Distribución y taxonomía de Pyrgophorus platyrachis (Caenogastropoda: Hydrobiidae), en el Sistema de Maracaibo, Venezuela
}

\author{
Mario Nava ${ }^{1}$, Héctor Severeyn ${ }^{1} \&$ Nakary Machado ${ }^{2}$ \\ 1. Laboratorio de Sistemática de Invertebrados Acuáticos, Departamento de Biología, Facultad Experimental de \\ Ciencias, Universidad del Zulia, Apartado postal 4011 Maracaibo, Venezuela; mariolesternava@yahoo.com, hectorse- \\ vereyn@yahoo.com \\ 2. Laboratorio de Oceanografía, Departamento de Biología, Facultad Experimental de Ciencias, Universidad del Zulia, \\ Apartado postal 4011 Maracaibo, Venezuela; nakmachado@gmail.com
}

Recibido 12-X-2010. C Corregido 02-II-2011. Aceptado 01-III-2011.

\begin{abstract}
Distribution and taxonomy of Pyrgophorus platyrachis (Caenogastropoda: Hydrobiidae) in the Sistema de Maracaibo, Venezuela. The presence of a microgastropod identified as Potamopyrgus sp. was detected previously in the Maracaibo System; nevertheless, a detailed morphological analysis identified this snail in other genera. The objective of this work is to update the distribution and taxonomy of Pyrgophorus platyrachis in the Maracaibo System, Venezuela in samples obtained between 2001 and 2009. The presence of hundreds of individuals of $P$. platyrachis were observed in the estuary, indeed in the localities of the Gran Eneal lagoon (4 111 snails), Peonías lagoon (229 snails), Punta Capitán Chico (758 snails), San Francisco (2 517 snails), Curarire (240 snails), Apon River mouth (173 snails), Ojeda City (240 snails), Bachaquero (128 snails) and Tomoporo de Agua (385 snails). We performed a taxonomical analysis, and emphasized in ecological aspects, such as the distribution of the species and habitat features, as near vegetation and type of associated sediment. We found three morphotypes of the species, one smooth, another with spiral striations and the other with spines. Smooth morphotype was exclusive of the Gran Eneal lagoon, Peonías lagoon, Punta Capitan Chico and Apon River mouth localities, whereas the other two morphotypes were found together in the remaining localities. According to our detailed anatomical and taxonomical analysis we propose a synonymy between $P$. platyrachis and the other species described like Pyrgophorus parvulus and Pyrgophorus spinosus. Rev. Biol. Trop. 59 (3): 1165-1172. Epub 2011 September 01.
\end{abstract}

Key words: Pyrgophorus platyrachis, distribution, taxonomy, Maracaibo System, Venezuela.

La familia de gasterópodos Hydrobiidae comprende especies de talla media a pequeña, operculados y principalmente de agua dulce o salobre. Para su identificación a nivel de especie, es de suma importancia la forma de la rádula y el órgano reproductor, tanto femenino como masculino. La concha es una característica secundaria en la taxonomía de esta familia, ya que es relativamente variable y polimórfica (Thompson 2004). La familia incluye aproximadamente 200 géneros y unas 1000 especies, donde conchas idénticas pueden pertenecer a subfamilias y géneros no relacionados (Thompson 2004).
A este respecto se ha planteado que los géneros Pyrgophorus Ancey 1888 y Potamopyrgus Stimpson 1865, son sinónimos, mientras que otros autores opinan que ambos se encuentran restringidos geográficamente (Rangel 2000). La similitud en la escultura de la concha en el género Pyrgophorus con respecto a Potamopyrgus, es lo que ha llevado a que sean considerados sinónimos, sin embargo las especies de Pyrgophorus son mucho más pequeñas, con cinco o menos vueltas y de sutura poco profunda (Zaranco et al. 1997). Harrison (1984) menciona los puntos taxonómicos clave que diferencian los dos géneros, Pyrgophorus 
presenta espinas que incluyen verdadero material de la concha, mientras que Potamopyrgus sólo presenta proyecciones de la epidermis; por ello Kabat \& Hershler (1993) destacan que éstos ya no son considerados sinónimos.

Pyrgophorus ha sido reportado en hábitats estuarinos y de agua dulce de Texas y Florida (EEUU), México y el Caribe. En los Estados Unidos se han reportado dos especies, muy similares a la especie introducida de Nueva Zelanda Potamopyrgus antipodarum Gray 1843, pero estas difieren en su anatomía reproductiva (Pérez et al. 2004), ya que Pyrgophorus presenta en su pene una proyección trilobulada (Thompson 2004), mientras que Potamopyrgus carece de este tipo de apéndices accesorios (Climo 1974).

Pyrgophorus está distribuido en la región sur de Norte América y al norte de Sur América, siendo los caracoles adultos de naturaleza dioica. Ninguna especie de este género en Norte América presenta parecido con $P$. antipodarum a nivel de las características de la concha (Zaranco et al. 1997).

Los ejemplares de ambos géneros forman densas poblaciones; $P$. antipodarum es partenogénico, lo que fácilmente le permite generar nuevas poblaciones; en el parque nacional Yellowstone (EEUU) se han reportado densidades poblacionales extremadamente elevadas, e incluso compite con especies nativas (Pérez et al. 2004).

La extensión geográfica de Pyrgophorus coronatus Pfeiffer 1840, es muy amplia según Pérez \& López (2003). En Venezuela previamente se ha reportado la presencia de Potamopyrgus (Pyrgophorus) parvulus Guilding 1828, para el área de Río Yumarito en Boquerón y Potamopyrgus (Pyrgophorus) valenciae Preston 1909, como fósil para el Lago de Valencia (Baker 1930).

Es importante destacar, que desde hace más de 35 años se conoce la presencia de un microgasterópodo en las aguas estuarinas del Sistema de Maracaibo. Rodríguez (1973) y Severeyn \& Rodríguez (2000) lo denominaron Potamopyrgus sp., por lo tanto no se identificó la especie. Después de las extensas recolecciones de este gasterópodo y de realizar un detallado análisis de la morfología de su concha, así como un minucioso estudio de su órgano genital masculino (pene), se ha llegado a la conclusión de que se trata de otro género, muy similar, pero anatómicamente diferente.

El objetivo de este estudio es realizar una redescripción del micromolusco, perteneciente al género Pyrgophorus, incluyendo su denominación específica correcta. También, se hace una detallada descripción de su órgano genital masculino y sus variantes a nivel de la concha; adicionalmente, se aportan datos de su distribución y hábitats dentro del Sistema de Maracaibo, Venezuela.

\section{MATERIALES Y MÉTODOS}

El tiempo de recolecta y número de ejemplares utilizados en el presente trabajo pueden observarse en el Cuadro 1, los mismos son procedentes del Sistema de Maracaibo, un estuario ubicado en la zona occidental de Venezuela (Rodríguez 2000); y específicamente de nueve localidades (Fig. 1): la Laguna de Gran

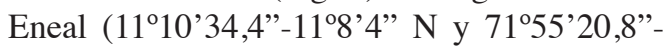
$71^{\circ} 53$ '30” W), Laguna Las Peonías (1046'20”-

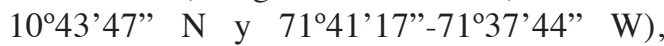
Punta Capitán Chico (1042'22"'-1041'59" N y 71'35'22"-71'34'51" W), San Francisco

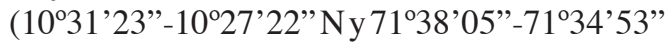
$\mathrm{W})$, Curarire $\left(10^{\circ} 11^{\prime} 45,7^{\prime \prime}-10^{\circ} 12 ' 23,0{ }^{\prime \prime} \mathrm{N}\right.$ y 71 $51^{\prime} 19,1^{\prime \prime}-71^{\circ} 50 ' 37,4$ " W), Ciudad

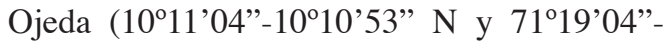
$71^{\circ} 18^{\prime} 47^{\prime}$ 'W), Desembocadura del Río Apón

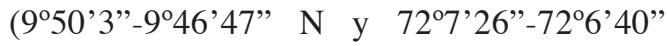
W), Bachaquero (9 $58^{\prime} 43^{\prime \prime}-9^{\circ} 53$ ' 40 " N y $71^{\circ} 14^{\prime} 17^{\prime \prime}-71^{\circ} 05^{\prime} 59^{\prime \prime}$ W) y Tomoporo de Agua

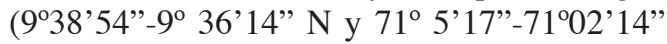
W). Las muestras de sedimento, recolectadas con una draga Ekman, se pasaron a través de un tamiz de $600 \mu \mathrm{m}$. Los ejemplares que se obtuvieron vivos fueron identificados por medio de una lupa estereoscópica siguiendo la recomendación de Thompson (2004), y preservados en alcohol etílico al 70\%. Los ejemplares se encuentran depositados en la sección de invertebrados acuáticos del Museo de Biología 


\section{CUADRO 1}

Número de ejemplares recolectados y tiempo de muestreo por cada localidad

TABLE 1

Number of snails collected and sampled time for every station

\begin{tabular}{lcccc}
\multicolumn{1}{c}{ Localidad } & $\begin{array}{c}\text { Ejemplares } \\
\text { observados }\end{array}$ & $\begin{array}{c}\text { Ejemplares machos } \\
\text { observados }\end{array}$ & $\begin{array}{c}\text { Ejemplares } \\
\text { recolectados }\end{array}$ & $\begin{array}{c}\text { Mes(es) y año de recolecta } \\
\text { Laguna de Gran Eneal }\end{array}$ \\
\hline Laguna Las Peonías & 229 & 2 & 4111 & $* 07 / 2008-06 / 2009$ \\
Punta Capitán Chico & 50 & 2 & 229 & $* 06-10 / 2009$ \\
San Francisco & 100 & 2 & 758 & $* 02-12 / 2008$ \\
Curarire & 240 & 2 & 2517 & $03 / 2001$ \\
Ciudad Ojeda & 240 & 2 & 240 & $* 05-09 / 2009$ \\
Río Apón & 60 & 2 & 240 & $* 06-10 / 2009$ \\
Bachaquero & 128 & $* *$ & 173 & $08-10 / 2009$ \\
Tomoporo de Agua & 385 & $* *$ & 128 & $01 / 2007$ \\
& & & 385 & $02 / 2001$
\end{tabular}

*=El guión (-) marca el período muestreado de manera mensual.

**=No se observaron los ejemplares machos ya que son muestras que reposan en el MBLUZ antes de la reidentificación de la especie.

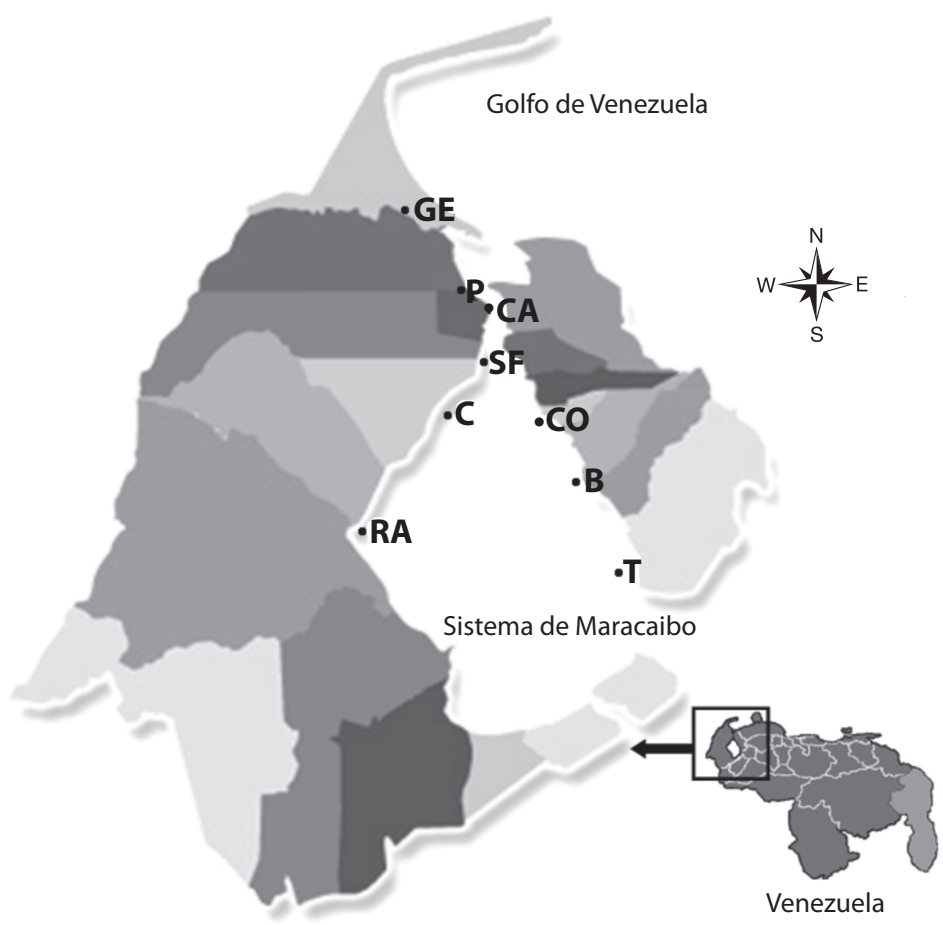

Fig. 1. Localidades de muestreo donde se encontró P. platyrachis dentro del Sistema de Maracaibo. GE=Gran Eneal, $\mathbf{P}=$ Peonías, $\mathbf{C A}=$ Capitán Chico, $\mathbf{S F}=$ San Francisco, $\mathbf{C}=$ Curarire, $\mathbf{C O}=$ Ciudad Ojeda, $\mathbf{B}=$ Bachaquero, $\mathbf{R A}=$ Desembocadura Río Apon y $\mathbf{T}=$ Tomoporo de Agua.

Fig. 1. Sampling stations where P. platyrachis was found in the Maracaibo System. GE=Gran Eneal, P=Peonías, CA=Capitán Chico, $\mathbf{S F}=$ San Francisco, $\mathbf{C}=$ Curarire, $\mathbf{C O}=$ Ojeda City, $\mathbf{B}=$ Bachaquero, $\mathbf{R A}=$ Apon River mouth y $\mathbf{T}=$ Tomoporo de Agua. 
de la Universidad del Zulia (MBLUZ), Maracaibo, Venezuela, bajo los números 2504 (Gran Eneal), 2505 (Las Peonías), 2506 (Punta Capitán Chico), 2507 (San Francisco), 2508 (Curarire), 2509 (Ciudad Ojeda), 2510 (Desembocadura Río Apón), 2511 (Bachaquero) y 2512 (Tomoporo de Agua).

También, se realizó una descripción taxonómica general de la especie, tanto de la concha como del órgano reproductor de los organismos machos que se obtuvieron vivos. Igualmente se observaron las variaciones morfológicas sólo a nivel de la escultura de la concha (lisa, estriada y/o espinosa) para la identificación de los morfotipos.

\section{RESULTADOS}

Descripción taxonómica: Los ejemplares del Sistema de Maracaibo se identificaron como $P$. platyrachis, se caracterizan por poseer un opérculo pauciespiral, la concha es de textura lisa, o enroscada con proyecciones espinosas triangulares en el borde superior de las vueltas. La misma es elongada y cónica, cuyo tamaño oscila entre 2 y $4 \mathrm{~mm}$ de largo. El periostraco es color marrón claro, aunque en algunos ejemplares correspondientes a la desembocadura del Río Apón es oscuro. Presenta de cinco a seis vueltas con una sutura marcada entre ellas.

El pene presenta de tres a siete papilas a lo largo del margen derecho, aunque en la mayoría de los especímenes estudiados se observaron cuatro, presenta una proyección con papilas (de uno a cuatro) cercana al extremo final del margen izquierdo; y en el extremo se presenta una pequeña mancha oscura (Fig. 2).

\section{Variación morfológica y distribución:}

De ahí que, se encontraron tres morfos a nivel de la escultura de la concha. El primero corresponde a especímenes principalmente lisos, pertenecientes a las localidades de la laguna de Gran Eneal, laguna Las Peonías, Capitán Chico y desembocadura del Río Apón. El segundo, de escultura estriada, y el tercero de escultura estriada-espinosa (Fig. 3), los cuales se encontraron juntos en las localidades de San Francisco, Curarire, Ciudad Ojeda, Bachaquero y Tomoporo de Agua, aunque en las otras

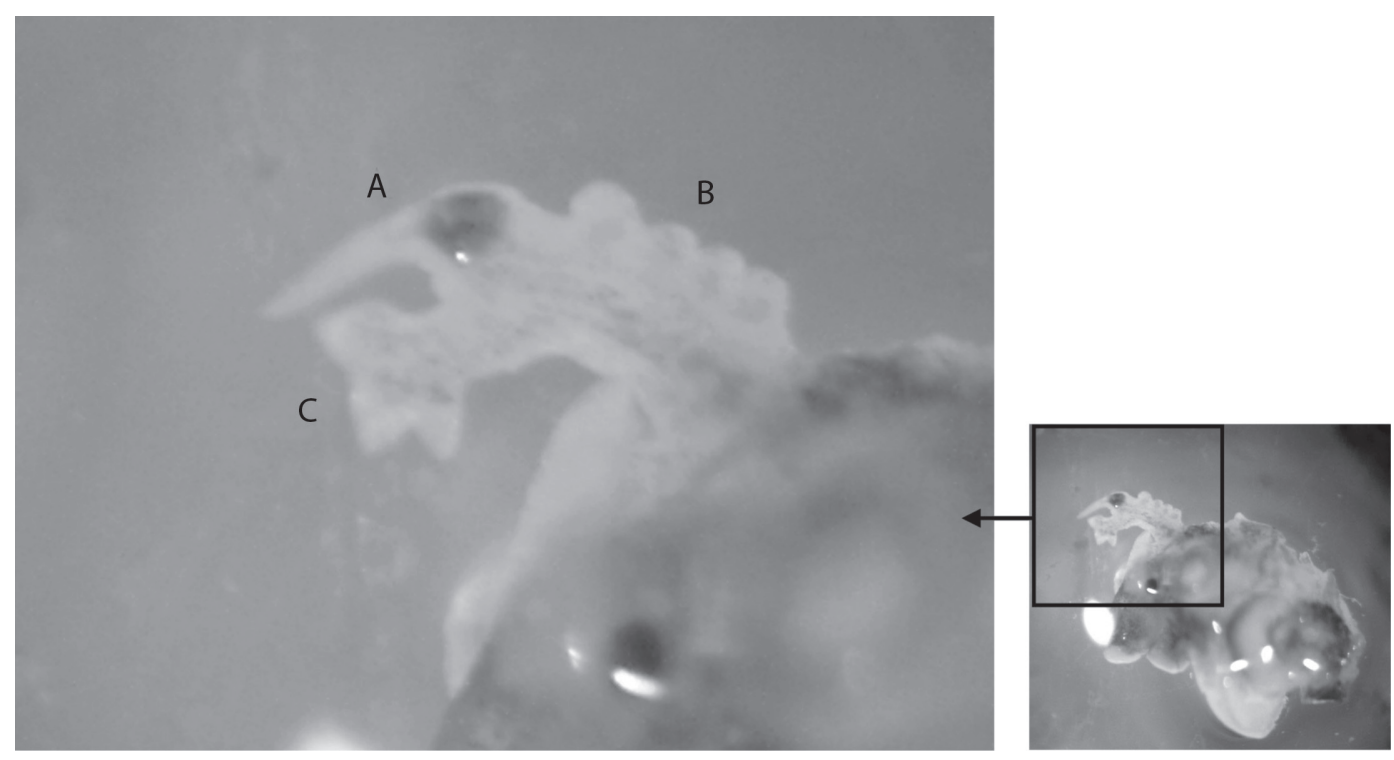

Fig. 2. Pene de P. platyrachis. (A) Pigmentación; (B) Papilas; (C) Proyección con tres papilas. Fig. 2. P. platyrachis penis. (A) Pigmentation; (B) Papillae; (C) Projection with three papillae. 

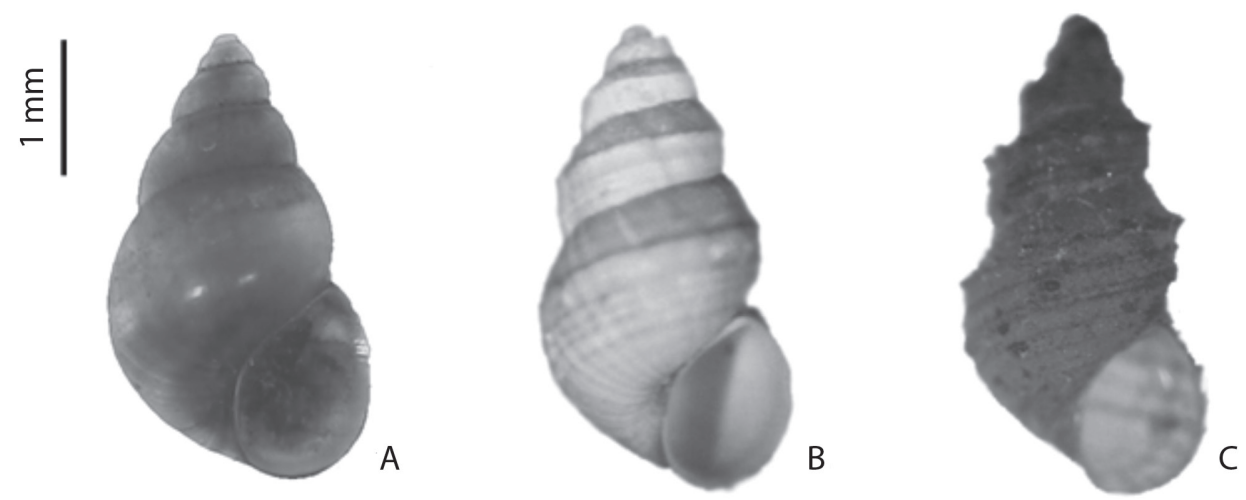

Fig. 3. P. platyrachis del Sistema de Maracaibo: (A) Morfotipo liso; (B) Morfotipo estriado; (C) Morfotipo espinoso. Fig. 3. P. platyrachis: (A) Smooth morphotype; (B) Spiral threads morphotype; (C) Spiny morphotype.

estaciones se encontraron individuos espinosos que no presentaron estrías y las tenían en una proporción muy baja. Estos morfotipos solo difieren en características a nivel de sus conchas, ya que la anatomía del cuerpo del animal y los órganos sexuales analizados (penes) es idéntica en todos ellos.

$P$. platyrachis presenta una amplia distribución dentro del estuario, desde la Laguna de Gran Eneal y Las Peonías al norte, hasta la localidad de Tomoporo de Agua al Sur-Este. Estos ambientes se caracterizan por presentar: sedimentos finos (arenosos finos a semifangosos) y algunos de ellos zonas de manglar cercanas. Cabe destacar que los organismos de la desembocadura del Río Apón se encontraron en su totalidad asociados a las raíces de Pistia stratiotes L. 1753.

\section{DISCUSIÓN}

Las características halladas a nivel de la escultura de la concha entre los ejemplares de las localidades de la Laguna de Gran Eneal, Las Peonías, Punta Capitán Chico y Río Apón, difieren con respecto a las obtenidas en Curaríre, San Francisco, Ciudad Ojeda, Bachaquero y Tomoporo, que tienen escultura principalmente lisa para el primer grupo de localidades, y enroscada-espinosa para el segundo. Existe un fuerte polimorfismo en las especies de Pyrgophorus, en donde se han señalado dos morfotipos básicos, uno liso y otro espinoso, existiendo una variación e intensidad de la escultura entre ambas formas (Pérez \& López 2003). Esta variación de morfotipos, podría tener significado ecológico, que quizás se debe a una adaptación para evitar la depredación selectiva (Covich 1976, Vermeij \& Covich 1978), o bien, a otras características ambientales específicas.

Por otra parte, la anatomía del pene de los individuos analizados en este estudio, coincide con lo descrito por Baker (1930) para P. parvulus, tanto en la pigmentación como en la forma que presentan, éste reporta tres papilas del lado derecho y una proyección trilobulada del lado izquierdo, aún cuando posee un número exacto de papilas, esto no es una particularidad que pueda indicar que se trata de otra especie distinta. Smith \& Drousseau (1996), reportaron la presencia de P. parvulus en Saint John, en las Islas Vírgenes, destacando que a nivel de la anatomía del pene, sus ejemplares no se diferencian de lo descrito por otros autores para P. platyrachis, P. parvulus y Pyrgophorus chagresensis Morrison 1946. Este autor destaca que la única característica que podría separar 
sus ejemplares con respecto a $P$. platyrachis, es la posición de las espinas en la concha, ya que cuando están presentes, se encuentran en la última vuelta de la concha del animal, mientras que en sus ejemplares se localizaron a nivel de la espira; estas diferencias podrían deberse a respuestas de plasticidad fenotípica a nivel de la concha. Burch \& Tottenham (1980) ilustran la anatomía de $P$. platyrachis, la cual muestra concordancia con los ejemplares encontrados en el Sistema de Maracaibo.

Rangel (2000), encuentra $P$. coronatus en México, destaca en su discusión una comparación entre especies como $P$. parvulus, $P$. platyrachis y Pyrgophorus spinosus Call \& Pilsbry 1886, y enfatiza que la revisión de este género es bastante confusa y compleja, aunque para él, existen diferencias que separan a $P$. coronatus de las otras especies.

Pyrgophorus ha sido ampliamente reportado en Norte América y el Caribe, destacan los trabajos de Baker (1930), Burch \& Tottenham (1980), García-Cubas et al. 1990; Brown (1991), Rangel (2000), Bass (2003), Pérez \& López (2003), Thompson (2004), Pérez et al. (2004) y Cañete et al. (2004). También ha sido considerablemente revisado, debido a que entre la segunda mitad del siglo XIX y la primera mitad del siglo XX se han descrito numerosas formas del mismo como especies válidas que posiblemente sólo sean variaciones de origen genético o ambiental (Pérez \& López 2003).

Las especies de Pyrgophorus son invasoras en potencia. En Hawai se ha reportado $P$. coronatus como una especie no endógena, la cual se introdujo desde el Caribe (Englund 2002). Es importante hacer mención del carácter endógeno del género Pyrgophorus en Sudamérica y el Caribe, en vista de su presencia en estado fósil en dichas regiones (Wesselingh \& Salo 2006), lo que confirma su presencia en el pasado remoto, y por lo tanto descarta la idea de ser una especie exótica o ajena al Sistema de Maracaibo.

En Venezuela se ha revelado la presencia de $P$. parvulus, en el Parque Nacional Archipiélago Los Roques y $P$. coronatus en el Lago de Valencia, estado Aragua, (Tello 1975); más específicamente para el Sistema de Maracaibo, Rodríguez (1973, 2000), señala la distribución de Potamopyrgus sp. en Punta Capitán Chico y $7 \mathrm{~km}$ al norte de Maracaibo. Rojas \& colaboradores (2006) encuentran especímenes de $P$. spinosus en el sistema estuarino Río Limón (Gran Eneal y Sinamaica), en donde es una de las especies dominantes de dicho sistema. Con los nuevos datos que aporta el presente estudio, se amplía el ámbito de distribución de $P$. platyrachis (anteriormente identificado como Potamopyrgus sp.) dentro del Sistema de Maracaibo. Tomando como base los estudios malacológicos realizados en dicho Sistema (Severeyn \& Rodríguez 2000, Severeyn et al. 2003, Nava 2005, Matusalén 2005) P. platyrachis esta restringida a las zonas estuarinas de baja salinidad del sistema; aun cuando es catalogada como una especie de agua dulce, ésta presenta cierta tolerancia a aguas salobres (Thompson 2004).

Es importante destacar que $P$. platyrachis, esta distribuido principalmente en humedales costeros del Estuario de Maracaibo o zonas cercanas a estos (Medina \& Barboza 2006), lo cual puede deberse a la alta productividad de este tipo de ambientes y a la capacidad que podrían presentar estos caracoles para detectar fuentes de alimento de origen animal y vegetal por quimiorecepción, e inclusive a sus hábitos detritívoros tal como a sido reportado en el género Potamopyrgus (Haynes \& Taylor 1984). Los ejemplares fueron obtenidos en su totalidad en zonas submareales, asociados a fondos blandos, lo cual concuerda con lo reportado por otros autores que incluso relacionan estos organismos con macrófitas (Talbot \& Ward 1987, Thompson 2004), lo cual en parte se confirma con los individuos encontrados en la desembocadura del Río Apón que estuvieron en su totalidad asociados a P. stratiotes.

Analizando los datos taxonómicos aportados por Baker (1930), Burch \& Tottenham (1980), Smith \& Drousseau (1996), Thompson (2004) y éste estudio, se considera que $P$. parvulus, $P$. spinosus y P. platyrachis son sinónimos de una misma especie, tomando en cuenta 
incluso el alto polimorfismo que presenta el animal a nivel de su concha.

\section{AGRADECIMIENTOS}

A Lisandro Moran por ayudar de manera incondicional en los muestreos de campo de algunas localidades incluidas en este estudio. A la División de Investigación de la Facultad Experimental de Ciencias de La Universidad del Zulia por el préstamo del vehículo para las salidas de campo hacia algunas de las localidades estudiadas y a dos revisores anónimos los cuales aportaron excelentes sugerencias al presente manuscrito.

\section{RESUMEN}

Algunos autores han planteado que los géneros Pyrgophorus Ancey 1888 y Potamopyrgus Stimpson 1865, son sinónimos, mientras que otros opinan que ambos se encuentran restringidos geográficamente. La similitud en la escultura de la concha, es lo que ha llevado a que sean considerados sinónimos, sin embargo las especies de Pyrgophorus son mucho más pequeñas, con cinco o menos vueltas y de sutura poco profunda. El objetivo de este trabajo es dar a conocer la distribución y taxonomía del gasterópodo de agua dulce Pyrgophorus platyrachis en el Sistema de Maracaibo, estado Zulia, Venezuela. Durante muestreos efectuados entre 2001 y 2009 se detectó la presencia de cientos de individuos de P. platyrachis en las localidades de la laguna de Gran Eneal, laguna Las Peonías, Punta Capitán Chico, San Francisco, Curarire, desembocadura del Río Apón, Ciudad Ojeda, Bachaquero y Tomoporo de Agua. Por lo tanto, se realizó un análisis taxonómico de los ejemplares obtenidos, destacando su distribución y la presencia de tres morfotipos: liso, enroscado y espinoso; el primero limitado a la Laguna de Gran Eneal, las Peonías, desembocadura del Río Apón y Punta Capitán Chico, mientras que los otros dos se encuentran juntos en las localidades restantes. Además, se propone la sinonimia entre P. platyrachis y otras especies descritas como Pyrgophorus parvulus y Pyrgophorus spinosus.

Palabras clave: Pyrgophorus platyrachis, distribución, taxonomía, Sistema de Maracaibo, Venezuela.

\section{REFERENCIAS}

Baker, H.B. 1930. The Mollusca collected by the University of Michigan-Williamson expedition in Venezuela. Occas. Paper. Mus. Zool. Univ. Mich. 210: 1-95.
Bass, D. 2003. A survey of freshwater macroinvertebrates in Tobago. Living World, J. Trin. \& Tob. Field Nat. Club 2003: 64-69.

Brown, K.M. 1991. Mollusca: Gastropoda, p. 285-314. In J.H. Thorp \& A.P. Covich (eds.). Ecology and classification of North American freshwater invertebrates. Academic, San Diego, EEUU.

Burch, J.B. \& J.L. Tottenham. 1980. North American freshwater snails: species list, ranges, and illustrations. Walkerana 1: 81-215.

Cañete, R., M. Yong, J. Sánchez, L. Wong \& A. Gutiérrez. 2004. Population dynamics of intermediate snail hosts of Fasciola hepatica and some environmental factors in San Juan y Martinez municipality, Cuba. Mem. Inst. Oswaldo Cruz 99: 257-262.

Climo, F.M. 1974. Description and affinities of the subterranean molluscan fauna of New Zealand. New Zeal. J. Zool. 1: 247-284.

Covich, A. 1976. Recent changes in molluscan species diversity of a large tropical lake (Lago de Peten, Guatemala). Limnol. Oceanogr. 21: 51-59.

Englund, R.A. 2002. The loss of native biodiversity and continuing nonindigenous species introductions in freshwater, estuarine, and wetland communities of Pearl Harbor, Oahu, Hawaiian Islands. Estuaries 25: 418-430.

García-Cubas, A., F. de la Llata, L. González \& M. Reguero. 1990. Moluscos de la laguna Mecoacán, Tabasco, México: Sistemática y ecología. Anales Inst. Cienc. Mar. Limnol. UNAM 17: 1-30.

Harrison, A.D. 1984. Redescription of Pyrgophorus parvulus (Gastropoda:Hydrobiidae) from St. Vincet, St. Lucia, and Granada, West Indies. Proc. Acad. Nat. Sci. Phila 136: 145-151.

Haynes, A. \& B.J. Taylor. 1984. Food finding and food preference in Pomatopyrgus jenkinsi (E. A. Smith, 1884) (Gastropoda: Prosobranchia). Arch. Hydrobiol. 100: 479-491.

Kabat, A. \& R. Heshler. 1993. The Prosobranch snail family Hydrobiidae (Gastropoda: Rissooidea): Review of classification and supraspecific taxa. Smithsonian Institution, Washington D.C., EEUU.

Matuzalen, J. 2005. Distribución y abundancia de macroinvertebrados bentónicos intermareales en playas de alta energía de la costa Sureste del Golfo de Venezuela. Tesis de Licenciatura, Universidad del Zulia, Maracaibo, Venezuela. 
Medina, E. \& F. Barboza. 2006. Lagunas costeras del Lago de Maracaibo: Distribución, estatus y perspectivas de conservación. Ecotrópicos 19: 128-139.

Nava, M. 2005. Macroinvertebrados bentónicos intermareales en playas arenosas de alta energía en el Noroeste del Golfo de Venezuela. Tesis de Licenciatura, Universidad del Zulia, Maracaibo, Venezuela.

Pérez, A.M. \& A. López. 2003. Listado de la malacofauna continental (Mollusca: Gastropoda) del Pacífico de Nicaragua. Rev. Biol. Trop. 51: 405-451.

Pérez, K.E., S.A. Clark \& L. Charles. 2004. Freshwater gastropod identification workshop "Showing your shells". Universidad de Alabama. Tuscaloosa, Alabama, EEUU. (También disponible en línea: www.cofc. edu/ dillonr/FMCSGastropodID.pdf)

Rangel-Ruiz, L. 2000. Estudio taxonómico de moluscos terrestres y dulceacuícolas de la región maya de México. I. Reserva de la biosfera de los Pantanos de Centla, México D.F., Universidad Juárez, Tabasco, México D.F.

Rodríguez, G. 1973. El Sistema de Maracaibo. IVIC, Caracas, Distrito Federal, Venezuela.

Rodríguez, G. 2000. El Sistema de Maracaibo. IVIC, Caracas, Distrito Federal, Venezuela.

Rojas, J., S. Theis, E. Ochoa, D. Pirela \& J. Rincón. 2006 Moluscos del sistema estuarino Río Limón, estado Zulia, Venezuela. Ciencia 14: 22-31.

Severeyn, H. \& G. Rodríguez. 2000. La fauna estuarina: Invertebrados, p. 199-224. In G. Rodríguez (ed). El Sistema de Maracaibo. IVIC, Caracas, Venezuela.

Severeyn, H., J. Delgado, A. Godoy \& Y. García de Severeyn. 2003. Efecto del derrame de petróleo del buque
Nissos Amorgos sobre la fauna macrobentónica del Golfo de Venezuela: cinco años después. Ecotrópicos 16: 83-90.

Smith, D. \& D. Brousseau. 1996. Survey of the freshwater mollusks of St. John, U.S. Virgin Islands, with descriptions and anatomical details of two species. Caribb. J. Sci. 32: 33-42.

Talbot, J.M. \& J.C. Ward. 1987. Macroinvertebrates associated with aquatic macrophytes in Lake Alexandrina, New Zealand. New Zeal. J. Mar. Freshwat. Res. 21: 199-213.

Thompson, F.G. 2004. Freshwater snails of Florida. A manual for identification. University of Florida, Gainesville, Florida, EEUU (También disponible en línea: www.flmnh.ufl.edu/malacology/fl-snail/ snails1.htm).

Tello, J. 1975. Catálogo de la Fauna Venezolana, VIII, Mollusca. Publicaciones de la comisión organizadora de la III conferencia de las Naciones Unidas sobre el derecho del mar, Caracas, Distrito Federal, Venezuela.

Vermeij, G.J. \& A.P. Covich. 1978. Coevolution of freshwaters gastropods and their predators. Am. Nat. 112: 833-843.

Wesselingh, F.P. \& J.A. Salo. 2006. A Miocene perspective on the evolution of the Amazonian biota. Scripta Geol. 133: 440-458.

Zaranco, D., D. Farara \& F.G. Thompson. 1997. Another exotic mollusk in the Laurentian Great Lakes: the New Zealand native Potamopyrgus antipodarum (Gray 1843) (Gastropoda, Hydrobiidae). Can. J. Fish. Aquat. Sci. 54: 809-814. 